\title{
Oesophageal cancer and experience with endoscopic stent intubation at St. Mary's Hospital, Nairobi
}

\author{
Ndonga A.K., MBChB, MMed (Surg), FCS (ECSA), Rucha M.R., MBChB, MMed (Surg), FCS (ECSA) \\ and Oigara R., MBChB, MMed (Surg), St Mary's Mission Hospital, Nairobi, P.O. Box 3409-00506, Nairobi, \\ Kenya \\ Corresponding author: Dr. Ndonga A.K., Department of Surgery, St Mary’s Mission Hospital, P.O. Box 3409- \\ 00506, Nairobi, Kenya, Email: andrewndonga@hotmail.com
}

\section{Abstract}

BACKGROUND: Oesophageal cancer often presents in advanced stages not amenable to surgical resection. In such patients, palliation of dysphagia remains the mainstay of management.

OBJECTIVES: To determine the burden of advanced oesophageal cancer and to document the experience with endoscopic metal stent intubation for its palliation

DESIGN: Retrospective evaluation of upper gastrointestinal (GI) endoscopy data and prospective study of stented patients.

SETTING: St Mary's Mission Hospital, Nairobi, Kenya.

SUBJECTS: Records of and patients who underwent upper gastrointestinal endoscopy and endoscopic stenting were evaluated .

RESULTS: Between July 2000 and July 2007, 8580 upper GI endoscopies were carried out, revealing tumour in 796 patients $(9.3 \%)$. Oesophageal cancer (both squamous cell and adenocarcinoma) accounted for 512 cases (64\%), $328(64 \%)$ being males. Only $49(9.6 \%)$ of the oesophageal tumours were deemed amenable to and underwent resection with curative intent. One hundred self expanding metal stents (SEMS) procedures were carried out over a 17-month period (March 2006 - July 2007). Of these procedures, performed under topical anaesthesia and injectable analgesics, only seven involved re-stenting. All patients were able to swallow immediately after. Procedure-related mortality was $2 \%$. Early procedure-related chest pain was a consistent feature $(100 \%)$. At follow-up, over half of the patients $(54 \%)$ had an objective weight gain before stabilizing or reducing as other tumour effects set in. The main study challenge was patient compliance with follow-up clinics.

CONCLUSION: Approximately one in ten patients referred for upper GI endoscopy had a tumour in this series. Oesophageal cancer was a common endoscopic finding and only a small percentage $(9.6 \%)$ was amenable to resection. Endoscopic stenting was found to be an affordable and effective minimally invasive outpatient procedure for palliation of dysphagia in non-resectable disease.

Key words: Oesophageal cancer, unsedated endoscopy, stenting (SEMS).

\section{Introduction}

Oesophageal carcinoma is the seventh most common malignancy worldwide and patient presentation is often late $(1,2)$. Unlike the rest of the gastrointestinal tract, the oesophagus has no serosal layer and advanced carcinomas penetrating the adventitia may therefore 
easily infiltrate adjacent organs (3). Evidence of distant spread or extensive local direct invasion into surrounding structures such as the tracheobronchial tree, aorta or vertebra by an oesophageal carcinoma precludes curative resection (3-5). Surgical resection for operable oesophageal cancer remains an option for a minority of cases with attendant five-year survival rates between $10 \%$ and $30 \%$ even in the best centres $(6,7)$. Despite protocols that combine use of surgery, radiation therapy and chemotherapy for lesions associated with submucosal spread, lymph-node involvement, and extension to surrounding structures (5), long-term survival is infrequent.

The major handicap in allocating and comparing treatment modalities for oesophageal cancer is the lack of precise preoperative staging (5). Modern staging methods include laparoscopy, bronchoscopy, computed tomography (CT), and endoscopic ultrasonography (4). The main role of CT in determining clinical tumour stage is the exclusion of $\mathrm{T} 4$ tumours demonstrated by the preservation of fat planes between an oesophageal cancer and adjacent structures. Magnetic resonance imaging (MRI) offers no significant advantage over CT (8). The efficacy of CT in preoperative staging of oesophageal carcinoma varies according to the location of the primary lesion, and it also has limitations in terms of staging lymph-node involvement (5), an important prognostic feature in resected oesophageal cancer (9). For many parts of Africa, these modalities are not routinely applied to preoperatively stage oesophageal carcinoma. Consequently huge disparities between pathologic and clinical stages ensue (5).

For the majority of patients for whom palliation forms the mainstay of care, the options include the use of pulsion and traction tubes, , feeding gastrostomy or jejunostomy and various other less-invasive procedures such as radiotherapy, laser therapy, photodynamic therapy and ethanol injection. Among these, endoscopic placement of self expanding metal stents (SEMS) has evolved as a main alternative minimally invasive option for palliation of the malignant dysphagia (10-12). In addition, there may be an improved survival benefit with stenting $(10,11)$. The practice of this procedure has been well documented since 1983 (13) but experience with the prostheses is limited in Kenya. This article evaluated the burden of oesophageal cancer at a mission hospital in Nairobi and documents the experience with SEMS for palliation of advanced malignant dysphagia.

\section{Materials and Methods}

The study was based on patients who underwent upper GI endoscopy at St Mary's Hospital in Nairobi. This 250-bed hospital serves mainly low-income and informal settlements' population within Nairobi and its environs.

To document the burden of oesophageal cancer, endoscopy unit records of patients who underwent upper GI endoscopies between July 2000 and June 2007 were retrieved. The diagnosis of oesophageal cancer was based on confirmed histology reports The burden was calculated as the rate of oesophageal cancer per 1,000 upper GI endoscopies.

From March 2006, endoscopic SEMS was offered to the patients with advanced disease. Because of prohibitive costs, most patients were deemed advanced on clinical grounds and were classified as such if three or more of the following criteria were fulfilled: (i). Progressive dysphagia of grade 2 and above for a period of 6 months or more, (ii) Progressive weight loss significant to the patient, (iii) Presence of persistent retrosternal pains not attributable to other causes, (iv) Circumferential tumour on endoscopy, with or without a remnant lumen, (v). Ultrasound evidence of abdominal spread in distal oesophageal tumour (below $30 \mathrm{~cm}$ on endoscopy) and (vi) Presence of a tracheooesophageal fistula.

Endoscopy was carried out using Olympus GIF 140 endoscope and unsedated technique. Stenting was carried out using partially coated AT\&M (Beijing, China, Released length $12 \mathrm{~cm}$, internal diameter $20 \mathrm{~mm}$ ) SEMS. Patients fasted for at least 6 hours prior to the endoscopy and received intramuscular analgesia at least half an hour prior. The procedure was explained 
and the oropharynx anaesthetized with $10 \%$ Lignocaine mucosal spray. An oral bite block was inserted with the patient in leftlateral position. The subject was then asked to gently swallow as the scope was advanced slowly. The procedure was carried out by a hospital surgeon assisted by one endoscopy technician. Under video control, a flexible-tip blunt guide-wire was advanced through any remnant oesophageal lumen or the centre of the tumour in complete obstruction. The surgeon confirmed the presence of guide-wire in the stomach by eliciting an affirmation from the patient, removed and started serial dilatations through the wire using cannulated dilators up to size 42 French gauge. The SEMS was then loaded, appropriate levels marked and the stent released into position. Proper placement was confirmed by immediate check endoscopy, also ensuring no residual oesophageal stricture /obstruction. The process lasted on average about 20 minutes and no further radiological tests were employed. The patients were assessed for ability to drink immediately after and also for post-operative complications. Ambulant patients were allowed home on the same day and any hospitalized patients discharged at the discretion of the attending physician. At followup, patients were assessed for complications and weight changes.

\section{Results}

Burden of oesophageal cancer: Eight thousand five hundred and eighty upper GI endoscopies were performed during the study period. These revealed tumours in 796 cases $(9.3 \%)$. There were 512 oesophageal (328 males, 184 females) 237 gastric (142 males, 95 females) and 47 other tumours (Figure 1). The calculated burden of oesophageal cancer among patients referred for endoscopic evaluation of upper gastrointestinal symptoms was 0.596 per 1000 endoscopies.

Of the 512 oesophageal tumours, only 49 $(9.6 \%)$ were operated for early disease. Other patients had intubations, had too advanced tumour for any active intervention or were referred for care elsewhere.
Figure 1: Frequency of upper GI tumours

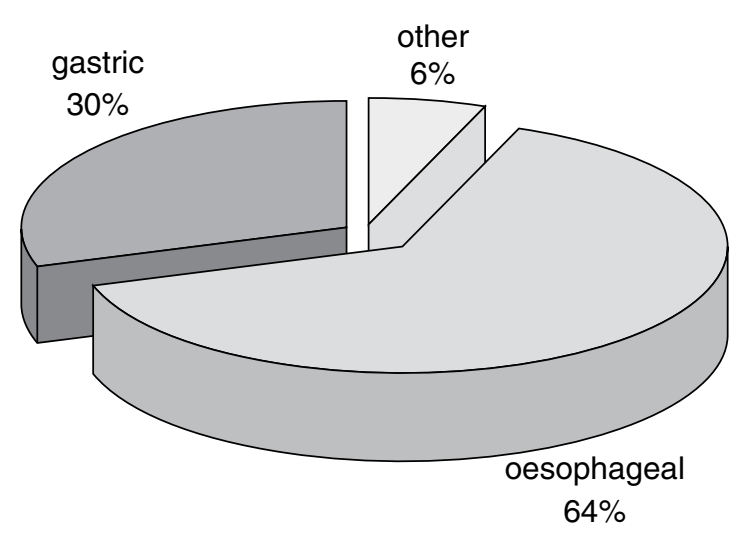

Experience with endoscopic stent placements: The self expanded metal stents became available in our institution in March 2006. The first 100 patients who underwent the procedure (over a 17-months period) formed the prospective arm of the study. Patient ages ranged from 33 to 105 years (mean 63 years). The proportion of patients less than 50 years was $18 \%$.

The tumour was in the upper third (above $22 \mathrm{~cm}$ ) in $17 \%$, middle third in $51 \%$ and lower third (below $30 \mathrm{~cm}$ ) in $32 \%$ of cases. Only 13 patients $(13 \%)$ were hospitalized prior to the procedure mainly due to long home distance, severe dehydration, or other co-morbidities such as aspiration pneumonia. The proportion of patients weighing $50 \mathrm{~kg}$ or less at endoscopy was $71 \%$.

Ninety three stents were new while seven were re-stents. All patients were able to swallow immediately after the procedure. Two patients died within 24 hours and this was attributed to oesophageal haemorrhage and gastric perforation in a severely tight distal oesophageal malignant stricture respectively. All patients experienced pain (early chest pain) over and above that due to the initial illness. Follow-up was inadequate. Sixty six patients attended the first scheduled post-discharge clinic. By the seventh month, only nine patients were still on follow-up (Table 1). The longest duration of follow-up by the close of the study was 11 months. These data excluded 11 patients who indicated they would prefer to be followed up in facilities near their homes. 
For those from whom follow-up data were available, tumour overgrowth / undergrowth was documented in 10 patients necessitating a second stent placement in seven patients who presented with renewed dysphagia.

Body weight increased in 36 patients, remained the same in 13 and reduced in 17 patients at the $1^{\text {st }}$ post-discharge clinic.

Table 1: Follow-up pattern of post SEMS patients

\begin{tabular}{lcc}
\hline $\begin{array}{l}\text { Months post- } \\
\text { discharge }\end{array}$ & $\begin{array}{c}\text { Number lost to } \\
\text { follow-up }(\mathrm{n}=82)\end{array}$ & $\%$ loss \\
\hline one & 16 & 19.5 \\
three & 41 & 50.0 \\
five & 58 & 70.7 \\
seven & 73 & 89 \\
nine & 76 & 92.6 \\
eleven & 79 & 96.3 \\
\hline
\end{tabular}

\section{Discussion}

Oesophageal cancer was the most common endoscopically-diagnosed tumour. Although it accounted for $64 \%$ of upper GI tumours, only a small percentage $(9.6 \%)$ had early-stage disease. As experienced elsewhere, many patients presented with advanced dysphagia, when treatment options are limited and palliation is of prime concern $(1,2,5,14,15)$. We found unsedated endoscopic stenting for palliation to be acceptable, safe and practical in our patients as has been experienced elsewhere and adapted a similar fashion for more expedient stenting $(12,17)$. The cost of using an expandable metal stent has been found more favourable when compared with other forms of palliative therapy that often require repeated procedures resulting in a prolonged in-patient hospital stay $(11,18)$. Comparative cost with Celestine intubation in our hospital puts SEMS at 70\% the cost of Celestine intubation (Unpublished data). In addition, patients were ambulant, could feed immediately after and had no wounds to be taken care of. The version of stent used in this institution is obtained at a cost about $1 / 7$ that of other western-quoted stents (1).
Procedure-related mortality at $2 \%$ was acceptably low as in Western studies where mortality figures from $0 \%$ to $7 \%$ have been quoted (19). In our study, this was attributed to tumour hemorrhage and gastric perforation. In a large review of over 2000 patients treated with oesophageal tubes, pulsion tubes were associated with a mortality of $11 \%$ due to hemorrhage among other complications (12). In a series of 59 SEMS patients (12) there was 1 post-op death due to cardiac arrest in a patient with bronchopleural fistula. SEMS has also been practiced in one centre in Kenya with low mortality and good reduction of dysphagia (20).

Early post-procedure chest pain occurred in all patients as in other studies (1). Stent overgrowth and undergrowth occurred at a rate of $10 \%$ as a late complication. Additional complications reported in literature and not captured in our series include stent migration. Cordero et al reported a single stent migration in their series (12). This could be attributed to the partial coating of stent used in our study, allowing for a firmer grip on the oesophageal wall.

Majority of the patients, as expected from advanced stage of disease, had low body weight at presentation. Body weight was however sustained or even increased within a month after the procedure in $49(74 \%)$ of the 66 patients who presented at their first clinic appointments. This is attributable to the minimally-invasive and ambulatory nature of the procedure. There is an obvious psychological boost when a patient is able to swallow immediately after the 'surgery'. Progressive weight loss despite the procedure occurred in 17 of the follow-up group. The weight reduction even without worsening of dysphagia may denote a poor prognostic feature predicting earlier demise. A study conducted in a larger Kenyan hospital revealed that majority of oesophagectomy patients continue to lose weight after surgery (21).

This study had several limitations. Staging relied mainly on clinical criteria. Radiological adjuncts including the use of CT scanning was unavailable to the clinicians unless outsourced at an extra cost burden to the patients who were mostly the poor from the informal settlements of Nairobi. This limits the external validity of our 
data. The follow-up was inadequate and thus a limit to provision of the one main outcome of palliation - dysphagia free survival. The median time to death following stent placement has been reported in the West to be between 77 days and 168 days, while one series reported a prolonged survival time (median 318 days) following stent placement in patients receiving concurrent radiation and chemotherapy (1). In another review, average survival was 4.2 months (12). Our loss to follow-up rates seem to follow the expected survival trend. More information should be sought in a targeted fashion to document whether indeed the follow-up pattern can be the proxy for survival pattern. Where follow-up is complete, survival ranges from four weeks to twelve months (12). Concomitant availability and use of CT would lend further credence to more widespread use of objective simple clinical staging criteria as entry point to SEMS management of oesophageal carcinoma at St. Mary's hospital.

\section{Conclusion}

SEMS offers a safe and affordable alternative palliation for malignant oesophageal dysphagia. Unsedated endoscopy and SEMS intubation is easy and available with equivalent outcomes at a low-cost setting. The loss to follow-up challenge must be addressed to unravel the true impact of endoscopic stenting in our locality.

\section{Acknowledgements}

Dr. R. White and endoscopy unit staff of Tenwek hospital for initiating us to stenting technique. The administration and staff of St Mary's mission hospital for cooperation extended to the endoscopy team enabling the study to be undertaken. Dr. Saidi Hassan and Prof Asad Raja of the Aga Khan University Hospital Nairobi on their useful hints for the published article. Findings reported here were presented at the $58^{\text {th }}$ ASEA $/ 7^{\text {th }}$ COSECSA Conference, Mombasa on 7th December 2007.

\section{References}

1. Lee S.H. The Role of oesophageal stenting in the nonsurgical management of oesophageal strictures. Brit. J. Radiol. 2001; 74: 891-900.

2. Quinn K.L., and Reedy A. Oesophageal cancer: Therapeutic approaches and nursing care. Semin. Oncol. Nurs. 1999; 15: 17-25.

3. Marcus V.A., Redston M. and Cullen J.B. Pathology of malignant oesophageal neoplasms. Oesophageal Surgery $2^{\text {nd }}$ Edition $(02002$ Churchill Livingstone. 678-682.

4. Wong J. and Law S.Y.K. Esophagogastrectomy for carcinoma of the esophagus and gastric cardia. Mastery of Surgery. $4^{\text {th }}$ Edition (C) 2001 Lippincott Williams \& Williams. Pg 813.

5. Krasna M.J., Sonett J.R. and Gamliel Z. Video-Assisted approaches to staging of oesophageal carcinoma. Oesophageal Surgery $2^{\text {nd }}$ Edition $@ 2002$ Churchill Livingstone. Pg. 695-696.

6. Siewert J.R., Hoelscher A.H. and Dittler H.J. Preoperative staging and risk analysis in oesophageal carcinoma. Hepatogastroenterol. 1990; 37: 382-387.

7. Wijnhoven B.P.L., Tran K.T.C., Esterman A.,et al. An Evaluation of prognostic factors and tumour staging of resected carcinoma of the esophagus. Ann. Surg. 2007; 245: 717-725.

8. Rice T.W. Diagnosis and staging of oesophageal carcinoma. Oesophageal Surgery $2^{\text {nd }}$ Edition (2002 Churchill Livingstone. Pg 686-687.

9. Reynold J.V., Muldoon C., Hollywood D., et al. Long-term outcomes following neoadjuvant chemoradiotherapy for oesophageal cancer. Ann. Surg. 2007; 245: 707-716.

10. Bethge N., Sommer A., von Kleist D., et al. A prospective trial of self-expanding metal stents in the palliation of malignant oesophageal obstruction after failure of primary curative therapy. Gastrointest. Endosc. 1996; 44: 283-286.

11. Knyrim K., Wagner H.J., Bethge N., et al. A controlled trial of an expansile metal stent for palliation of oesophageal obstruction due to inoperable cancer. $N$. Engl. J. Med. 1993; 329: 1302-1307.

12. Cordero J.A. and Moores D.W.O. Oesophageal Intubation. Oesophageal Surgery $2^{\text {nd }}$ Edition (C2002 Churchill Livingstone. Pg 903-907. 
13. Frimberger E. Expanding spiral-a new type of prosthesis for the palliative treatment of malignant oesophageal stenosis. Endoscopy. 1983; 15: 213-214.

14. Ogendo S.W.O. Surgery of the oesophagus: A Nairobi experience. East Afr. Med. J. 1993; 70: 307-309.

15. Law S. and Wong J. Current management of oesophageal cancer. J. Gastrointest. Surg. 2005; 9: 291-310.

16. Baron T.H. Expandable metal stents for the treatment of cancerous obstruction of the gastrointestinal tract N. Engl. J. Med. 2001; 344: 1681-1687.

17. O'Connor J.J. Office esophagogastroduodenoscopy. Int. Surg. 2007: 92; 32-33.

18. Nicholson D.A., Haycox A., Kay C.L., et al. The cost effectiveness of metal oesophageal stenting in malignant disease compared to conventional therapy. Clin. Radiol. 1999; 54: 212-215.

19. Adam A., Ellul J., Watkinson A.F., et al. Palliation of inoperable oesophageal carcinoma: A prospective randomised trial of laser therapy and stent placement. Radiol. 1997; 202: 344-348.

20. White R.E., Mungatana C., Harewood G.C., et al. Self-expanding metal stents as sole treatment for advanced oesophageal cancer. Dis. Esophagus. 2004; 17 (S1): A53

21. Ogendo S.W.O. Weight change post oesophagectomy for carcinoma of oesophagus. East Afr. Med. J. 2007; 84: 271-278. 